\title{
THE EFFECT OF A ROUGH CORE-MANTLE BOUNDARY ON PKKP ${ }^{1}$
}

\author{
D.J. DOORNBOS \\ Vening Meinesz Laboratory, University of Utrecht, Lucas Bolwerk 6, 3512 EG Utrecht (The Netherlands)
}

(Received June 3, 1979; revised and accepted July 5, 1979)

Doornbos, D.J., 1980. The effect of a rough core-mantle boundary on PKKP. Phys. Earth Planet. Inter., 21: $351-358$.

Scattering by a slightly-rough core-mantle boundary (CMB) with small-scale radial variations of up to a few hundred metres, has been an attractive (though non-unique) interpretation of at least part of the precursors to PKIKP. Here it is shown that a slightly-rough CMB has an observable effect on PKKP as well, if the signal-to-noise ratio is sufficiently high. The effect may be observed as precursive arrivals and is due to back-scattering PK $\wedge$ KP at CMB. This work was prompted by observations by Chang and Cleary at LASA of "PKKP" and precursors from the Novaya Zemlya explosions. NORSAR data from several source regions are presented here; small-scale radial variations of 100-200 metres are inferred from these data, although in some regions the CMB appears to be much smoother. On the other hand, the LASA data are anomalous and suggest much larger topography in the sampled region of the CMB. Both large- and small-scale topography must be dynamically produced, if current estimates of the viscosity of the lower mantle $\left(\sim 10^{22}\right.$ Poise $)$ are correct.

\section{Introduction}

In the past, several publications have described the effect on core phases of velocity and density structure near the core-mantle boundary (CMB). Following theoretical advances, there has been a progression from investigations dealing with the simple planewave, plane-interface model, to models involving curvature of the interface and/or the wave front (which may be caused by a velocity gradient). Usually the interface has been taken to be smooth, but it is becoming apparent that deviations from a smooth CMB may effect short-period core phases, and that this effect is observable in certain circumstances. In this respect, interest was focused mainly on precursors to PKIKP in the approximate distance range $125-140^{\circ}$, following Haddon (1972) and Cleary and Haddon (1972). They suggested scattering at or above

1 NORSAR publication No. 264. the $\mathrm{CMB}$ as an explanation of the precursors, and this has been amply verified. Scattering by both a slightly. rough $\mathrm{CMB}$ (radial variations up to a few hundred metres) and a slightly-heterogeneous lower mantle (relative variations in physical parameters up to a few percent) produces the energy level that is observed in most of the PKP precursors (Doornbos, 1978), although there are exceptions, in particular, a number of large precursors, requiring strong scattering above the CMB, have been observed at NORSAR.

In a recent paper, Chang and Cleary (1978) have drawn attention to precursors to PKKP. Previously, they had been reported relative to the DF branch in the distance range $125-135^{\circ}$ along the back azimuth (Doornbos, 1974a). In that case the interpretation is similar to that of PKP precursors; it requires forward scattering at or near the CMB, upon entry into, or upon exit from the core. In connection with a remarkable observation at LASA, of PKKP and precursors from the Novaya Zemlya explosions at distances around $60^{\circ}$ along back azimuth, Chang and Cleary (1978) discussed the other possibility which is to 
obtain precursors to PKKP by backward scattering at the lower side of the CMB: PK $\rightarrow \mathrm{KP}$. Because of the maximum-time character of PKKP (BC and DF branches), this type of scattering will arrive prior to the main phase, or it will arrive at a distance where the main phase is absent. Back scattering by a rough interface may be equally efficient as forward scattering, whereas back scattering by volume heterogeneity is generally insignificant. Therefore, observations of this type would be important to answer the question of smoothness or roughness of the CMB. It should be noted that, although the back scattered sig. nal from a slightly-rough CMB is expected to be small (as it is required in a first order theory), ordinary reflections (of the $\mathrm{BC}$ and DF branches) are also small $(\sim 5 \%)$, so in this case scattered phases may not be negligible relative to the main phase. Figure 1 illustrates this point for one particular scattering model.

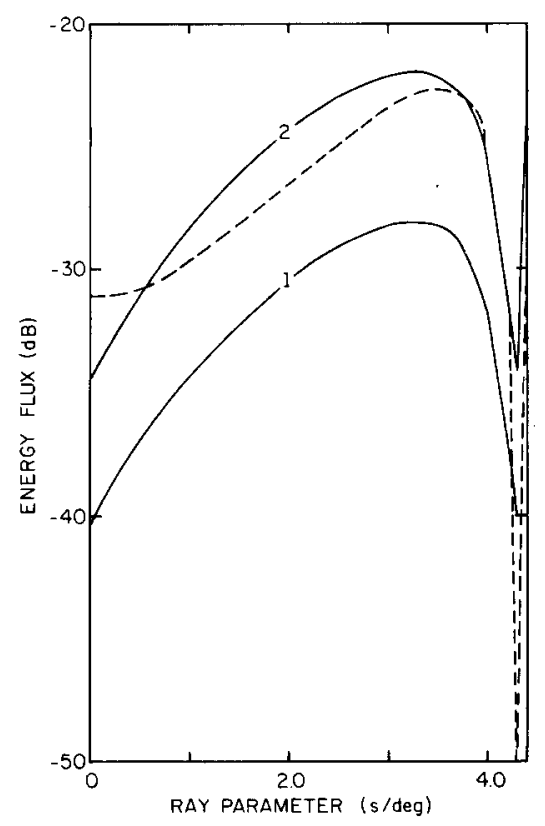

Fig. 1. Energy flux at $1 \mathrm{~Hz}$ through solid-liquid interface representing CMB in the PEMC-L01 model (see text). Dashed line: $\mathrm{P} \wedge \mathrm{P}$ reflection at lower side of the interface. Solid lines: $P \curvearrowright P$ scattered energy per unit of solid angle, in the direction of reflected $P$ (i.e. scattered ray parameter $p=$ incident ray parameter $p^{\circ}$ ). 1 : Rough interface with average height perturbation $\xi=100 \mathrm{~m}$ and scale length $\sigma=10 \mathrm{~km} ; 2$ : $\xi=100 \mathrm{~m}, \sigma=20 \mathrm{~km}$.
In Section 2 the aspects of scattering theory which are essential in explaining precursors to PKKP by a slightly-rough $\mathrm{CMB}$ are outlined. Observational evidence of the precursive phenomenon is obtained from NORSAR data. A comparison with computational results (Section 3 ) confirms the anomalous character of PKKP from the Novaya Zemlya explosions observed at LASA.

\section{Scattering analysis}

Anticipating, for the moment, a slightly-rough $\mathrm{CMB}$, causing scattering which is small compared to the incident wave field, we follow a conventional perturbation approach to replace the effect of the roughness by an equivalent dislocation on the (plane) interface. The procedure as applied to a solid-liquid interface has been described by Doornbos (1978) in some detail; here it suffices to give the essentials of the application to PKKP.

The equivalent dislocation depends on the incident wave field, the geometry of the interface, and the contrasts in density and elastic constants. At a solidliquid interface there is always a significant contrast in rigidity. At the CMB there is also a large density contrast. For these reasons, scattering by the CMB is comparatively effective. In the Earth, detailed specifications of the geometry of an interface (like the CMB) are rarely available, but for the present purpose it is sufficient to specify topography of the interface by an average-scale length and amplitude. Following previous work, this leads to the characterization of the interface by a random function. This function is usually represented by its spatial autocorrelation $r(x, y)$, whose Fourier transform is the wavenumber spectrum $R\left(k_{x}, k_{y}\right)$. For a Gaussian autocorrelation:

$$
\begin{aligned}
& r(x, y)=\xi^{2} \exp \left(-\frac{x^{2}+y^{2}}{\sigma^{2}}\right) \\
& R\left(\mathbf{k}_{h}\right)=\xi^{2} \pi \sigma^{2}\left(\exp -\frac{1}{4}\left|\mathbf{k}_{h}\right|^{2} \sigma^{2}\right) \\
& \mathbf{k}_{h}^{T}=\left(k_{x}, k_{y}\right)
\end{aligned}
$$

where $\xi$ and $\sigma$ represent the average amplitưde and scale length of the interface irregularity. In the far field expression for scattered energy (say at frequency $\omega$ and in location $\mathbf{x}$ ) the wavenumber spectrum con- 
veniently factors out to:

$E(\mathbf{x}, \omega) \sim \int_{S}\left|P\left(\mathbf{k}_{h}, \mathbf{k}_{h}^{0}, \omega\right)\right|^{2} R\left(\mathbf{k}_{n}-\mathbf{k}_{h}^{0}\right) d S$

where:

$P=\mathbf{C}^{T}\left(\mathbf{k}_{h}\right) \mathbf{L}\left(\mathbf{k}_{h}, \mathbf{k}_{h}^{0}, \omega\right) \mathbf{B}^{0}\left(\mathbf{k}_{h}^{0}\right)$

$\mathbf{B}^{\mathbf{0}}$ and $\mathbf{C}$ are Green's tensors for incident and scattered waves, respectively, and the connection matrix $\mathbf{L}$ depends on the contrasts in density and elastic constants across the interface. Plane wave approximations have been assumed in $\mathbf{B}^{\mathbf{0}}$ and $\mathbf{C}$, so the horizontal wavenumbers $\mathbf{k}_{h}^{0}$ and $\mathbf{k}_{h}$ depend on the scattering point (in $S$ ) and on the field point (in $\mathbf{x}$ ). To account for time dependence, the integrand in eq. 1 may be modified by an envelope function for which we take $\exp \left(-2 \gamma t^{2}\right)$, and $\gamma$ depends on the length of the incident signal. In the results to follow we have taken $\gamma=0.7$. Scale lengths which are relevant to the scattering may be determined by putting:

$\partial E / \partial \sigma\left(\omega_{0}\right)=0$

For the observed signals with frequencies around $1 \mathrm{~Hz}$, we have $\omega_{0}=2 \pi\left(\mathrm{rad} \mathrm{s}^{-1}\right)$, and the most effective scale lengths to generate precursors before the

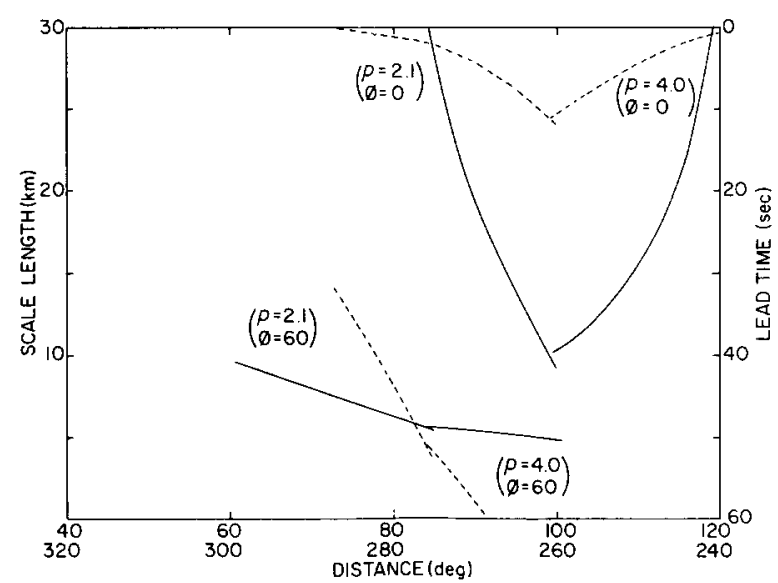

Fig. 2. Scattering from the $B C$ branch to the $B C$ branch of PKKP. Solid lines: Optimum scale length of a rough CMB in the PEMC-L01 model to generate PKKP precursors at $1 \mathrm{~Hz}$ versus epicentral distance. $p$ is ray parameter $(\mathrm{s} / \mathrm{deg})$ of incident ray, $\phi$ is deviation angle (deg.) between the diametral planes through the incident and scattered ray. Dashed lines: lead time relative to the BC branch of PKKP.

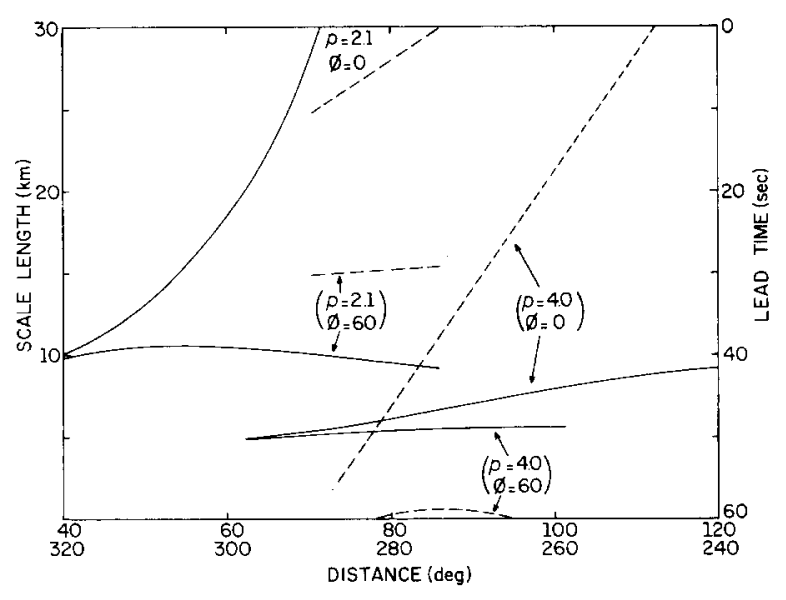

Fig. 3. Scattering from the $\mathrm{BC}$ branch to the $\mathrm{DF}$ branch of PKKP. Other details as in Fig. 2.

$\mathrm{BC}$ branch of PKKP have been plotted as a function of epicentral distance, for several combinations of incident and scattered ray directions (Figs. 2, 3). The corresponding time differences with PKKP are also shown. Computational results indicate that the scattered energy is maximum in a time interval just before PKKP (Section 3). Figures 2 and 3 then suggest that scale lengths of about $10-20 \mathrm{~km}$ are relevant here, and this happens to be just the range of scale lengths analysed previously with precursors to PKP (Doornbos, 1978). A meaningful comparison with these earlier results can thus be made.

\section{Data and computational results}

In order to infer characteristics of a rough interface, it is important to analyse the scattered waves in comparison to a suitable reference phase. Obviously, precursors to PKKP should be compared to PKKP itself whenever possible, since then the effects of lateral variations away from the $\mathrm{CMB}$, and of bias in the Earth model, will be minimal. Since the DF branch of PKKP is generally too small to be observed (as will be apparent later in this section, from Fig. 7), we relate scattered wave-energy to the $\mathrm{BC}$ branch of PKKP. Our main concern will therefore be with data in the approximate distance range $75-120^{\circ}$, although we shall also have occasion to discuss the data of 
Chang and Cleary (1978), around $60^{\circ}$. In the distance range $75-120^{\circ}$, short-period PKKP (BC) amplitudes are well determined by ray theory. Outside this range, diffraction effects become important and the amplitudes are sensitive to small details in the model. In this range, observable precursors most likely arrive at times just before the main phase, and to identify this phenomenon, short-period signals of simple appearance are required. This effectively limits the data to those from large arrays (NORSAR and LASA, in their stage of full deployment), and the events are preferably explosions or deep-focus earthquakes. Events analysed at the NORSAR array are listed in Table I. They are from several source regions in Southeast Asia and South America. We will now briefly outline our analysis procedure, present a number of typical examples, and compare the data to computational results for specified models of a rough CMB.

Bandpass filtered subarray signals having dominant frequencies around $1 \mathrm{~Hz}$, were combined to give average power versus time, and to give beampower versus time, for a range of $\mathrm{d} T / \mathrm{d} \Delta$ and azimuths around the expected value for PKKP. More detailed discussions of these techniques may be found, for example, in Doornbos and Husebye (1972) and King et al. (1975). Here we present only the beampower maxima ( $\mathrm{d} T /$ $\mathrm{d} \Delta$ and azimuth being variable). A full appreciation of the data requires their mapping in time, $\mathrm{d} T / \mathrm{d} \Delta$ and azimuth; for a number of selected events, that more extensive analysis is being conducted by Haddon (in prep.). Figure 4 shows results for a deep event near $106^{\circ}$. The data in this case were rather noisy (probably due to poor performance of one of the subarray channels), and a slightly-rough CMB cannot be inferred from the curve of average power. Beamforming improved the signal-to-noise ratio by about $10 \mathrm{~dB}$, and with the noise level now about 18 $\mathrm{dB}$ down from PKKP it is clear that energy is arriving prior to the main phase. As outlined in Section 2, scattered energy versus time can be computed for specified roughness of the CMB, and the dotted curve in Fig. 4 represents a dominant frequency of $1 \mathrm{~Hz}$, and an interface irregularity characterized by a scale length $\sigma=20 \mathrm{~km}$ and an average amplitude $\xi=200$ $\mathrm{m}$. In these and other computations in this paper, the PEM-C model of Dziewonski et al. (1975) was modified by the inclusion of a low-velocity zone at the base of the mantle, in accordance with model PEMCL01 (Doornbos and Mondt, 1979). The curve of scattered energy can be simply scaled to accommodate different amplitudes of irregularity, since scattered energy in the first order approximation is proportional to $\xi^{2}$. For the event in Fig. 4, a slightly rough CMB explains the precursive energy quite well. In the same figure, the array beams for PKKP and for $\mathrm{P}$ are displayed on top. Although the beam for PKKP may partly suppress the precursors (coming from slightly different directions), the essential features are still observable. It may also be observed that PKKP and P have the same polarity, which is as expected; the double Hilbert transform in PKKP caused by going through a pole (i.e. $\Delta>180^{\circ}$ ) and having a double

TABLE I

Earthquakes used in analysis of PKKP and precursors

\begin{tabular}{llccccc}
\hline Date & Source region & $\begin{array}{l}\text { Latitude } \\
\left({ }^{\circ} \mathrm{N}\right)\end{array}$ & $\begin{array}{l}\text { Longitude } \\
\left({ }^{\circ} \mathrm{E}\right)\end{array}$ & $\begin{array}{l}\text { Depth } \\
(\mathrm{km})\end{array}$ & $m_{b}$ & $\begin{array}{l}\text { Distance }\left(^{\circ}\right) \\
\text { to NORSAR }\end{array}$ \\
\hline 1973, Jan. 31 & Bonin Isl & 28.2 & 139.2 & 498 & 6.0 & 81.7 \\
1971 , July 27 & Peru-Ecuador & -2.7 & -77.4 & 135 & 6.3 & 91.5 \\
1972 , Jan. 12 & Western Brasil & -6.9 & -71.8 & 580 & 5.9 & 92.4 \\
1972, Oct. 1 & Philippines & 7.5 & 123.7 & 613 & 6.0 & 94.3 \\
1971 , April 8 & Southern Sumatra & -4.3 & 102.4 & 75 & 6.3 & 94.5 \\
1973, Oct. 25 & Argentina & -22.0 & -63.7 & 529 & 6.1 & 101.7 \\
1976, Jan. 23 & Flores Sea & -7.5 & 119.9 & 614 & 6.4 & 105.8 \\
1972, April 4 & Banda Sea & -7.4 & 125.6 & 377 & 6.6 & 108.4 \\
1971, Sept. 16 & Banda Sea & -5.9 & 130.7 & 115 & 6.2 & 109.4 \\
1971, Nov. 28 & Chile-Argentina & -29.8 & -69.5 & 105 & 5.9 & 111.1 \\
\hline
\end{tabular}




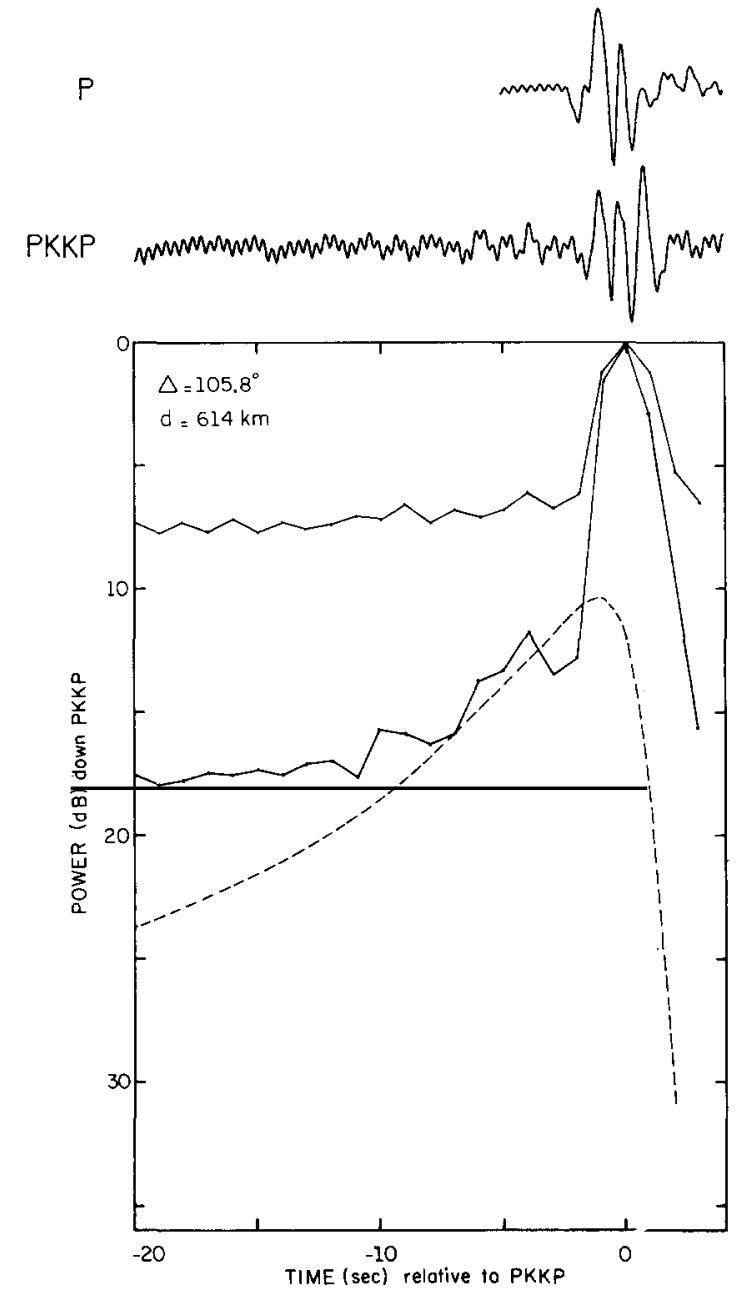

Fig. 4. Solid curves: average power and beampower in $\mathrm{dB}$ relative to $\operatorname{PKKP}(B C)$, versus time. Dashed curve: theoretical scattering power versus time, for dominant frequency $1 \mathrm{~Hz}$ and rough CMB characterized by scale length $\sigma=20 \mathrm{~km}$ and average amplitude $\xi=200 \mathrm{~m}$. Seismic traces on top are array beams for $\mathrm{P}$ and PKKP.

turning point (see e.g., Chapman, 1978), is compensated by the negative reflection-coefficient at the CMB.

Another example is shown in Fig. 5, which is for an event near $109^{\circ}$. The noise level here is extremely low, and the comparison with scattered energy curves demonstrates that the CMB is essentially smooth over the area sampled by PKKP from this event. Besides, this was the only event analysed which gave PKKP and $\mathbf{P}$ of opposite polarity (see the array beams on
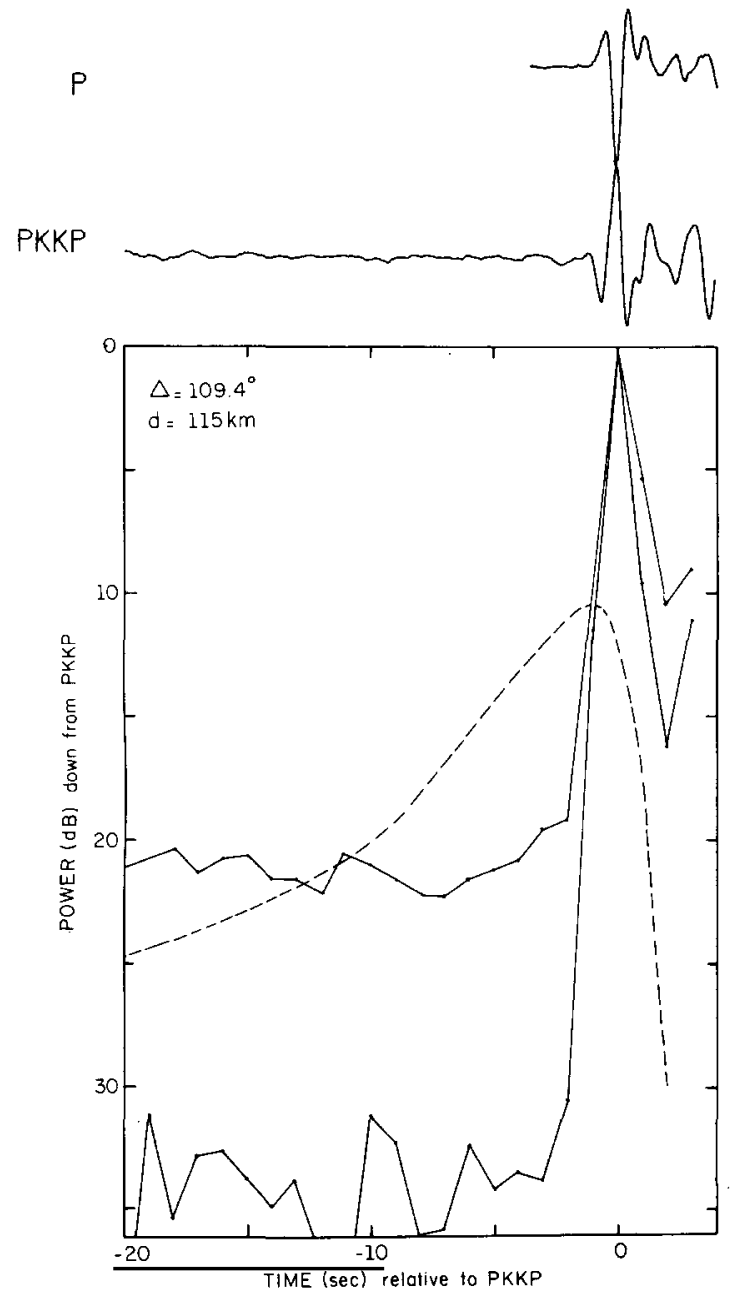

Fig. 5. Average power, beampower and theoretical scattering power versus time. Other details as in Fig. 4.

top). Since, at the source, the directions of wave propagation for PKKP and $\mathbf{P}$ differ by more than $30^{\circ}$, it is possible that these directions are opposite from a nodal plane; we have not checked this. A few other typical examples, with and without evidence of precursors to PKKP, are shown in Fig. 6.

In Fig. 7 we attempt to summarize the results. The data give maximum precursive energy for each event. Although this presentation unavoidably obscures certain details of the signals, we believe it provides a useful base for testing models of a rough CMB. Theoretical amplitudes at a frequency of $1 \mathrm{~Hz}$ are given for PKKP and PcP. The low amplitude of the DF branch 

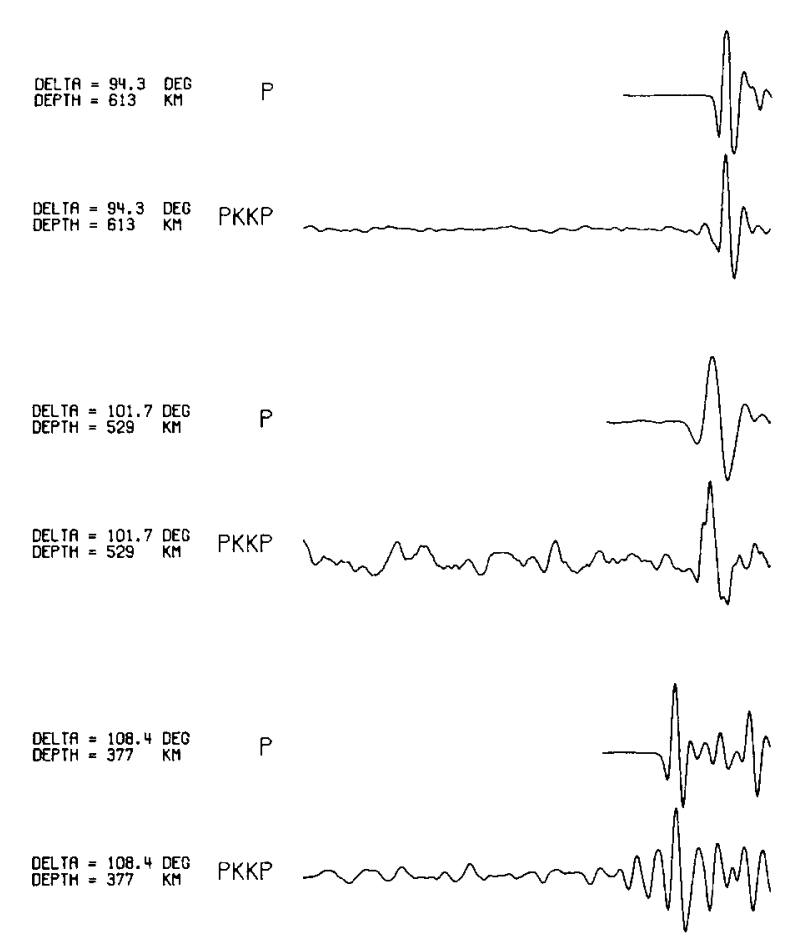

Fig. 6. Array beams for $P$ and PKKP from three events (Table 1). Length of PKKP traces is 24 seconds.

of PKKP is due, in part, to a low- $Q$ zone in the upper few hundred kilometres of the inner core, which has been inferred from short-period $P$ waves by several workers (see e.g., Anderson and Hart, 1978). In the amplitude calculations we have taken the $Q$-model of Doornbos (1974b). Theoretical scattering results correspond to a rough $\mathrm{CMB}$ with scale lengths of 10$20 \mathrm{~km}$, and average amplitude of $100-200 \mathrm{~m}$. Because most precursors have been observed within a few seconds before PKKP, we have given the theoretical results for maximum precursor energy (about one second before PKKP) and for energy at five seconds before PKKP. We are led to the conclusion that the precursive energy in our data is explained by a slightly-rough CMB with radial variations between $100-200 \mathrm{~m}$, although in some regions the interface appears to be much smoother. In this regard we mention that in most cases, the signal to noise ratio was not sufficiently high to resolve irregularities of the boundary with an amplitude $\xi<100 \mathrm{~m}$. The inferred roughness in the present work is quite compatible with the constraints set by precursors to PKP

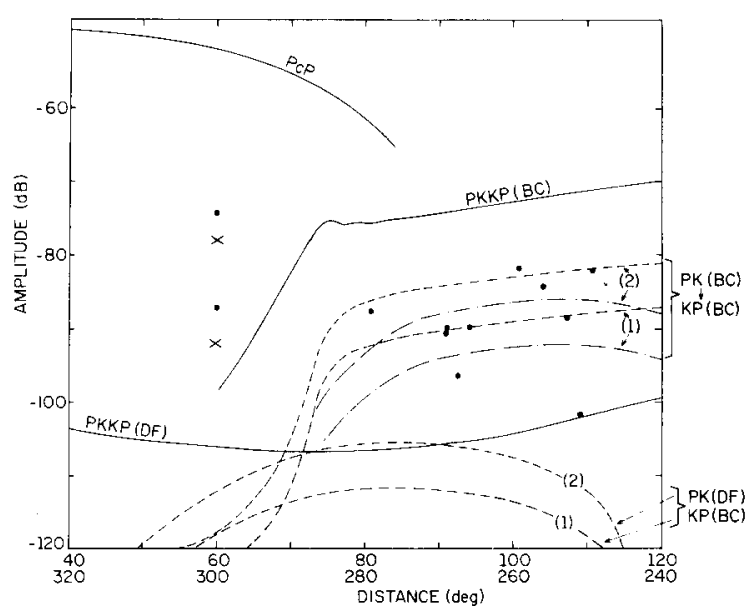

Fig. 7. Relative amplitudes of PKKP and PcP (solid lines, absolute amplitude level arbitrary), and scattering by a rough CMB (dashed lines); the frequency is $1 \mathrm{~Hz}$. (1) CMB irregularity with average amplitude $\xi=100 \mathrm{~m} ;(2) \xi=200 \mathrm{~m}$. Dashed lines, maximum precursor energy $(\sim$ one second before PKKP); dot-dashed lines, precursor energy five seconds before PKKP. Data (solid dots) at $\Delta>75^{\circ}$ are from NORSAR (Table I) and are taken relative to PKKP. Data (solid dots) in $\Delta=60^{\circ}$ represent the Novaya Zemlya event of 27 October 1973 observed at LASA and are taken relative to $\mathrm{PcP}$. Crosses are theoretical amplitudes for reflection at dipping $\mathrm{CMB}$; reflection from $\mathrm{BC}$ branch to $\mathrm{BC}$ branch (upper cross) and from DF branch to $\mathrm{BC}$ branch (lower cross).

$(\xi \simeq 200 \mathrm{~m}$, with other options, i.e. structure above the CMB).

In Fig. 7 we also give the relative amplitudes of "PKKP" and the maximum precursor as displayed by Chang and Cleary (1978) for the Novaya Zemlya explosion of 27 Oct., 1973. Peak-to-peak amplitudes were related to $\mathrm{PcP}$ taken from a figure of Massé et al. (1974). Although the resulting data in Fig. 7 are undoubtedly very crude, the amplitude difference with the ordinary PKKP(DF) phase is so large that other explanations for the observed "PKKP" phase must be considered. In fact, it has already been noted by Chang and Cleary (1978) that, although the observed arrival time corresponds to PKKP(DF), the direction of approach did not, thus suggesting that it is a deflected $B C$ branch: $P K(B C) \rightarrow K P(B C)$. A similar suggestion is prompted by observed amplitudes of the precursors: $\mathrm{PK}(\mathrm{DF}) \rightarrow \mathrm{KP}(\mathrm{BC})$ and vice versa. Scattering by a slightly-rough CMB is clearly insufficient to produce the amplitudes. The other extreme 
possibility, corresponding to ordinary reflection coefficients by a dipping CMB, results in amplitudes which are close to the observed ones (Fig. 7). The implied model of the CMB is, however, embarassing, since the dip is about $7^{\circ}$ and in order to build up the amplitude, this dip must extend over many wavelengths. As a consequence, radial perturbations of CMB would exceed several $\mathrm{km}$ and this has not been observed from other data. Other models, involving strong scattering or diffraction by suitably oriented irregularities of the interface, have been considered by Haddon (in preparation). In either case, the Novaya Zemlya data seem to require large topographic features on the $\mathrm{CMB}$, and the region sampled by these data must be quite different from the regions sampled by all other data in this study.

\section{Concluding remarks}

The large contrast in rigidity and density across the $\mathrm{CMB}$, and the circumstance that scattered waves from a rough $\mathrm{CMB}$ may arrive as precursors to certain core phases, account for the remarkable fact that small-scale undulations of the boundary, with average amplitude within a few hundred metres, have an observable effect on the seismic records. Precursors to PKP are often observed on single stations, but their interpretation in terms of a rough CMB, though often adequate, is not unambiguous since scattering by volume heterogeneity in the mantle above the CMB may produce a similar effect. On the other hand, precursors to PKKP can be reliably extracted only with large arrays, and the phenomenon thus observed seems to be satisfactorily explained only by a rough CMB. Analysis of PKKP(BC) and precursors at NORSAR has led us to infer a rough CMB with average radial variations of $100-200 \mathrm{~m}$, although in some regions the boundary is found to be much smoother. This is fairly consistent with earlier inferences from many of the PKP precursors. However, "PKKP" and precursors from the Novaya Zemlya explosions observed at LASA, correspond to either strongly scattered waves (e.g. a mechanism as suggested by Haddon, in preparation), or the limiting case of reflections at a dipping boundary as suggested by Chang and Cleary (1978). In either case, large undulations of the boundary would be expected in the region sampled by these data. This result is quite different from what is inferred from the other data, and it suggests that whereas relief of the CMB is mostly small, this may not be the case in at least some regions.

Large undulations are clearly anomalous, but we remark that "small" undulations with amplitude $100-200 \mathrm{~m}$ are also significant in a physical sense. In the case of finite viscosity of the mantle, undulations of the CMB would tend to smooth out according to $\exp (-t / T)$, and a rough estimate of the decay time if $T \sim 2 \eta k /\left(g[\rho]_{+}^{-}\right)$, which follows from considerations similar to the postglacial rebound studies (e.g. Cathles, 1975). Here $k$ is a wavenumber characterizing the lateral scale of the undulations. For a scale length $\sigma=15 \mathrm{~km}$ we have $k=2 / 15 \mathrm{~km}$, and with $g=10$ $\mathrm{m} \mathrm{s}^{-2},[\rho]_{+}^{-}=4.4 \mathrm{~g} \mathrm{~cm}^{-3}, \eta=10^{22}$ Poise (Cathles, 1975), we find $T \sim 2 \cdot 10^{5} \mathrm{y}$. This time is small compared to the time of existence of the core, hence if current estimates of the viscosity of the lower mantle are correct, the undulations of the CMB must be dynamically produced.

\section{Acknowledgements}

I wish to thank Drs. Husebye and Haddon, who independently conducted a similar type of investigation, for cooperation and helpful comments. Part of this work was supported by NORSAR/NTNF.

\section{References}

Anderson, D.L. and Hart, R.S., 1978. $Q$ of the Earth. J. Geophys. Res., 83: 5869-5882.

Cathles, L.M., 1975. The viscosity of the Earth's mantle. Princeton University Press, Princeton, NJ.

Chang, A.C. and Cleary, J.R., 1978. Precursors to PKKP. Bull. Seismol. Soc. Am., 68: 1059-1079.

Chapman, C.H., 1978. A new method for computing synthetic seismograms. Geophys. J. R. Astron. Soc., 54: 481-518.

Cleary, J.R. and Haddon, R.A.W., 1972. Seismic wave scattering near the core-mantle boundary: a new interpretation of precursors to PKP. Nature (London), 240: 549-551.

Doornbos, D.J., 1974a. Seismic wave scattering near caustics: observations of PKKP precursors. Nature (London), 247 : $352-353$.

Doornbos, D.J., 1974b. The anelasticity of the inner core. Geophys. J. R. Astron. Soc., 38: 397-415. 
Doornbos, D.J., 1978. On seismic wave scattering by a rough core-mantle boundary. Geophys. J. R. Astron. Soc., 53: $643-662$.

Doornbos, D.J. and Husebye, E.S., 1972. Array analysis of PKP phases and their precursors. Phys. Earth Planet. Inter., 5: 387-399.

Doornbos, D.J. and Mondt, J.C., 1979. P and S waves diffracted around the core and the velocity structure at the base of the mantle. Geophys. J. R. Astron. Soc., 57: $381-395$.
Dziewonski, A.M., Hales, A.L. and Lapwood, E.R., 1975. Parametrically simple Earth models consistent with geophysical data, Phys. Earth Planet. Inter., 10: 12-48.

Haddon, R.A.W., 1972. Corrugations on the mantle-core boundary, or transition layers between inner and outer cores? Trans. Am. Geophys. Union, 53: 600 (abstract).

King, D.W., Haddon, R.A.W. and Husebye, E.S., 1975. Precursors to PP. Phys. Earth Planet. Inter., 10: 103-127.

Massé, R.P., Flinn, E.A., Seggelke, R.M. and Engdahl, E.R., 1974. PKIIKP and the average velocity of the inner core. Geophys. Res. Lett., 1: 39-42. 